\title{
Recent Reason for Hindering Medications for Perinatal Mental Disorders in Japan
}

\author{
Shunji Suzuki
}

\begin{abstract}
Background: We examined the recent reasons for hindering antipsychotic medications during pregnancy in Japan.

Methods: We retrospectively analyzed the medical charts of all women who gave birth after 22 weeks' gestation at Japanese Red Cross Katsushika Maternity Hospital from August 2016 to July 2017.

Results: Four pregnant women with mental disorders (three schizophrenia and one adjustment disorder) kept the interruption of medications under their partners' compulsion. All of their partners had a history of mental disorders (two schizophrenia, one anxiety disorder and one adjustment disorder).
\end{abstract}

Conclusion: In cases of pregnancy requiring mental health care, mental health care on partners seemed to be also needed.

Keywords: Pregnant women; Mental disorders; Interrupted antipsychotic medications; Partner

\section{Introduction}

Patients with serious mental disorders and their physicians face at least one challenge concerning the adverse effect on fetuses and/or infants caused by psychotropic medications during pregnancy and postpartum, because relapses seemed to be more frequent when antipsychotics are discontinued in these patients [1-3]. Ultimately, clinical decisions should be made on a case-by-case basis, weighing the risks to the mother in terms of symptom exacerbation and relapse if antipsychotic treatment is discontinued, and the potential risk on the fetuses and/or infants due to continued antipsychotic treatment [4]. Recently, the majority of antipsychotic medications have appeared to be relatively safe for use during pregnancy and breastfeeding $[4,5]$. In addition, accumulating research has

Manuscript submitted August 9, 2017, accepted August 25, 2017

Department of Obstetrics and Gynecology, Japanese Red Cross Katsushika Maternity Hospital, 5-11-12 Tateishi, Katsushika-ku, Tokyo 124-0012, Japan. Email:czg83542@mopera.ne.jp

doi: https://doi.org/10.14740/jocmr3155w shown that prenatal exposure of fetuses to maternal stress increases the risk for behavioral and mental health problems later in life [6]. Occasionally patients and their general practitioners interrupt antipsychotic medications; however, in most cases they can be resumed with the counseling by experts of perinatal mental health care based on the recent evidence [5, 6]. However, the effect of counseling sometimes did not appear well, and the mental status of the patients got worse due to the interrupted antipsychotic medications. In this study, we examined the recent trend in reason for the hindering antipsychotic medications in pregnant Japanese women.

\section{Methods}

The protocol for this analysis was approved by the Ethics Committee of the Japanese Red Cross Katsushika Maternity Hospital. In addition, informed consent was obtained from each subject before delivery.

To examine the reason for the hindering antipsychotic medications in pregnant Japanese women with mental disorders, we retrospectively analyzed the medical charts of all women who gave birth after 22 weeks' gestation at Japanese Red Cross Katsushika Maternity Hospital from August 2016 to July $2017(\mathrm{n}=1,925)$.

\section{Results}

A flow diagram of study inclusion is shown in Figure 1. During the study period, there were $58(3.0 \%)$ pregnant women with mental disorders diagnosed at pre-pregnancy by Japanese psychiatric specialists. Of the 58 women, $45(78 \%)$ had medications at pre-pregnancy. Of the 45 women, 16 (36\%) interrupted their antipsychotic medications depending on the individual and/or general practitioners' discretion during pregnancy. Of the 16 women, $12(75 \%)$ resumed their medications by our counseling after 2 - 16 weeks of the interruption, while the remaining four ( $25 \%$, three cases of schizophrenia and one case of adjustment disorder) kept the interruption of medications under their partners' compulsion. In the latter, all of the partners had a history of mental disorders (two schizophrenia, one anxiety disorder and one adjustment disorder), and the intimate partner violence (IPV) screenings using the modified violence against women screening (VAWS) [7], which is a Japanese screening instrument for IPV, in the first trimester were positive in all cases. 


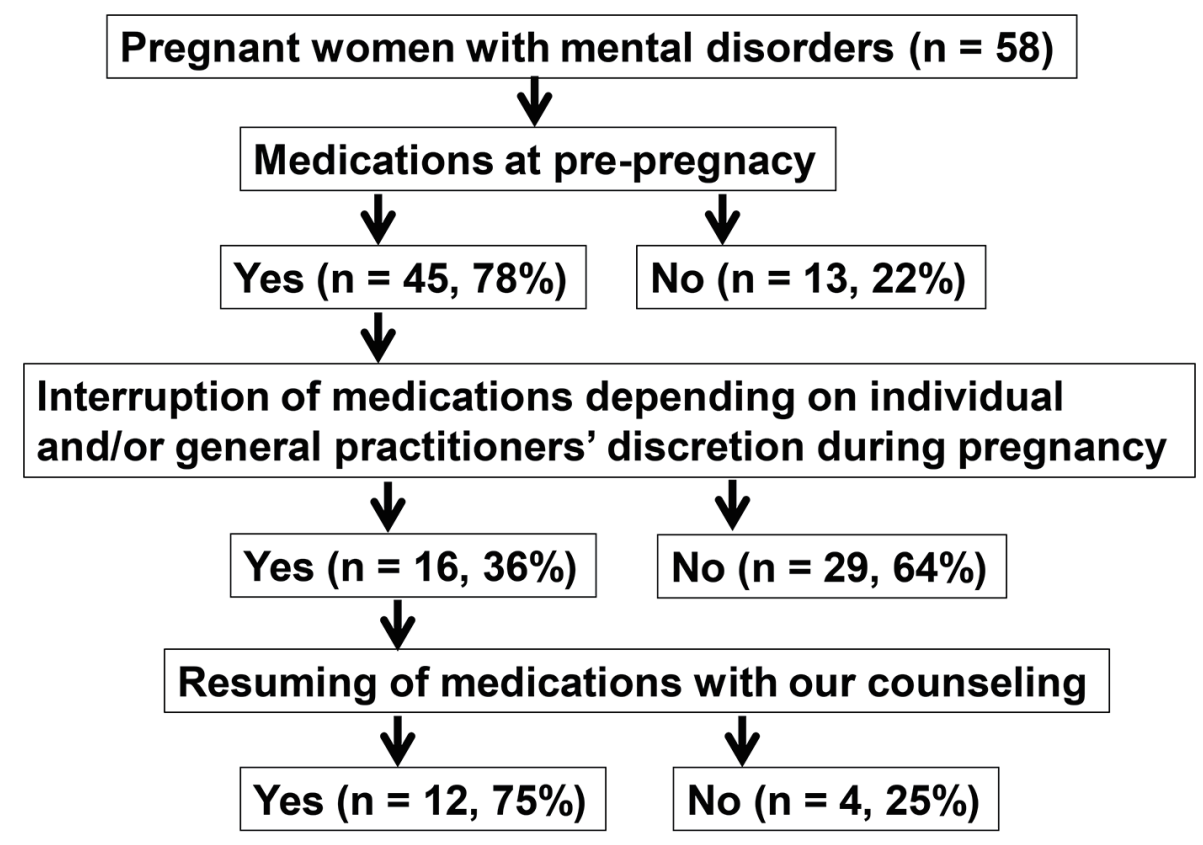

Figure 1. Flow diagram of study inclusion.

\section{Discussion}

It has been reported that most psychotropic medications are relatively safe to use during pregnancy and postpartum and that not using them when indicated for serious psychiatric illness poses a greater risk to both mothers and children [1-3, 5]. In recent years, this has been enlightened in the Japanese perinatal fields rapidly [8]. In the current experience, however, the mandatory intention (compulsion) of the patients' partners caused the deterioration of mental disorders during the pregnancy due to interrupt antipsychotic medications. Although we have believed that Japanese husbands are quiet and calm, IPV is also a serious social issue in Japan. For example, some previous studies in Japan highlighted that the rate of obvious IVP is about $4.1-5.4 \%$ in all population of pregnant women leading to the increased risk of mental disorders [7,9]. We may be worked hard and distressed by mental health care of mothers and children, while the care for their partners sometimes may be neglected. Once, for example, we had been concentrating on the mother's mental health care but unfortunately we missed child abuse by the father (unpublished case). We understand the small sample size of the current observation; however, the partners' attitudes and words seem to be the pressure of pregnant women.

In cases of pregnancy requiring mental health care, mental health care on partners seemed to be also needed. In addition, it is necessary to enlighten the safety of psychotropic medications during pregnancy and postpartum against the partners in Japan.

\section{Competing Interests}

The author declared that he has no competing interests regard- ing the publication of this paper.

\section{Author Contributions}

Shunji Suzuki performed all work of this paper.

\section{Reference}

1. Ifteni P, Moga MA, Burtea V, Correll CU. Schizophrenia relapse after stopping olanzapine treatment during pregnancy: a case report. Ther Clin Risk Manag. 2014;10:901904.

2. Cohen LS, Altshuler LL, Harlow BL, Nonacs R, Newport DJ, Viguera AC, Suri R, et al. Relapse of major depression during pregnancy in women who maintain or discontinue antidepressant treatment. JAMA. 2006;295(5):499507.

3. Cohen LS, Nonacs RM, Bailey JW, Viguera AC, Reminick AM, Altshuler LL, Stowe ZN, et al. Relapse of depression during pregnancy following antidepressant discontinuation: a preliminary prospective study. Arch Womens Ment Health. 2004;7(4):217-221.

4. Robinson GE. Treatment of schizophrenia in pregnancy and postpartum. J Popul Ther Clin Pharmacol. 2012;19(3):e380-386.

5. Chisolm MS, Payne JL. Management of psychotropic drugs during pregnancy. BMJ. 2016;532:h5918.

6. Van den Bergh BRH, van den Heuvel MI, Lahti M, Braeken M, de Rooij SR, Entringer S, Hoyer D, et al. Prenatal developmental origins of behavior and mental health: The influence of maternal stress in pregnancy. Neurosci Biobehav Rev. 2017. 
7. Kataoka Y, Yaju Y, Eto H, Horiuchi S. [Domestic violence against women during pregnancy]. Nihon Koshu Eisei Zasshi. 2005;52(9):785-795.

8. Suzuki S. Recent status of pregnant women with mental disorders at a Japanese perinatal center. J Matern Fetal
Neonatal Med. 2017:1-5.

9. Suzuki S, Yamada F, Eto M. Influence of intimate partner violence on mental status in Japanese women during the first trimester of pregnancy. J Matern Fetal Neonatal Med. 2017 (in press). 